\title{
A Full-scale Model to Predict Borehole Fluid Temperature with Groundwater Advection
}

\author{
Tengfei Cui
}

Shanshan Cai*
Haijin Guo
Ting Huang

\begin{abstract}
Ground source heat pump (GSHP) system is one of the major techniques that utilize shallow geothermal energy and borehole heat exchangers (BHEs) is the key component in GSHP system. Although most proposed analytical models for the thermal analysis of vertical BHEs take into account of the effects of either groundwater flow or axial heat conduction in the soil, the time scale of heat transfer in the BHEs is not well treated. In this study, an analytical full-scale model was developed to efficiently include the effects of the coupled transient heat transfer in the borehole, the axial heat conduction along the borehole and the effect of horizontal flow of groundwater. Results derived from the new model were compared with solutions of other traditional analytical and numerical models with the same configuration. The impacts of axial heat transfer and groundwater flow on the thermal response in the full time scale were also analyzed based on the proposed model.
\end{abstract}

\section{NTRODUCTI ON}

Ground source heat pump (GSHP) system is one of the major techniques that utilize shallow geothermal energy and borehole heat exchangers (BHEs) is the key component in the GSHP system (Somogyi, et al. 2017). Compared to horizontal boreholes, the vertical configurations are widely used in both commercial and residential buildings due to compact occupied areas and stable soil temperatures among different seasons. In order to estimate the heat transfer of vertical BHEs, different numerical (Kim, et al. 2010; Saner, et al. 2010), analytical (Diao, et al. 2004; Li and Lai 2012) and combined models (Hellström 1991; Yavuzturk 1999) have been proposed in the current literature, among which analytical methods are preferred because of the simple expression and fast computation.

The heat transfer in the vertical BHEs would be affected by several factors, such as the underground thermal properties and the variable building loads over years. Generally, it is recommended to consider the heat transfer procedure in two separated regions. One region is the space out of the borehole, where the heat transfer is usually treated as a transient process due to the fact that the heat exchanger is surrounded in a semi-infinite medium. The infinite line-source (ILS) model, finite line-source (FLS) model and the cylindrical source model are normally considered in this region (Zeng, et al. 2002). Compared to the other two methods, FLS model is more often used to include the effect of axial heat conduction along the borehole. The other region is the one in the borehole, which is filled with grouting material and U-shaped tube(s) with circulating medium. In the current literature, this region can be analyzed as steady state (including quasi steady state) due to the high aspect ratio (length/diameter $\approx 150 \mathrm{~m} / 0.1 \mathrm{~m}$ for example) and the low thermal capacity compared to the surroundings. Treating the U-shaped tube as a single pipe with equivalent diameter in 1-D is one of the simplest expressions. In order to include thermal influence between legs of U-shaped tube, researchers (Hellström 1991; Claesson and Hellström 2011) further developed different 2-D 
models based on tube, researchers (Hellström 1991; Claesson and Hellström 2011) further developed different 2-D models based on the line-source assumption and multipole method, respectively. A 3-D quasi steady state model was also proposed to include the effect of varied fluid temperature along the depth of the borehole (Diao, et al. 2004).

However, it would result in some issues by treating the heat transfer procedure in two separated regions. According to some researchers' work, the traditional line-source models would be valid for $\mathrm{t} \geq 5 r_{b}^{2} / a$, which is around 4 to 8 hours for a typical borehole (Eskilson and Claesson 1988). Therefore, the conventional line-source models are inappropriate for the short-term energy analysis of GSHPs. Although some analytical solutions are available to predict the short-term response of BHEs, most of them are based on the assumption of "equivalent-diameter", which simplifies the complicated geometry of the borehole to one composite region enclosed in a hollow cylinder (Javed 2011; Javed and Claesson 2011). This simplification makes it impossible to include the transient effect of both the grouting material and the fluid circulating in the U-shaped tube. With the same assumption, a full-scale model is proposed to cover a wide time range from few minutes to several decades (Javed 2011), but there exists a gap between the temperature profiles derived from the short- and long-term solutions and an arbitrary temperature shift is required at the "breaking time". Li (Li, et al. 2014) developed another full-scale model based on the idea of "matching asymptotic expansion" and this model is able to simulate the thermal response by considering different structural parameters and physical properties in the borehole. However, all these full-scale models do not take into account of the groundwater impact. Several researches indicate that in most cases, the impact of groundwater flow on the heat transfer of BHEs cannot be neglected. Groundwater flow promotes the heat transfer between the BHEs and surrounding, and in some cases, this enhancement may allow 16\% savings on the initial cost (Capozza, et al. 2013).

Although most proposed analytical models for the thermal analysis of vertical BHEs take into account of the effects of either groundwater flow or axial heat conduction in the soil, the time scale of heat transfer in the BHEs is not well treated. Therefore, in this paper, a new analytical full-scale model is developed to efficiently include the effects of the coupled transient heat transfer in the borehole, the axial heat conduction along the borehole and the horizontal flow of groundwater.

\section{FUNDAMENTAL THEORY}

This section provides a general review on the models that will be used as basis of the improved model. The schematic of vertical BHEs with groundwater flow is shown in Fig. 1. The assumptions are summarized as follows: i) the underground is homogeneous and composed of saturated soil with constant properties; ii) the surface temperature of the ground is constant; iii) the groundwater flows uniformly along x-axis with Darcy velocity $u_{d}$. In porous medium with groundwater flow, the 2-D heat transfer between the BHEs and the soil is mainly in the form of heat conduction and convection, as expressed in Eq. (1) (Nield and Bejan 2006).

$$
\rho C_{p} \frac{\partial T}{\partial t}+u_{d}\left(\rho C_{p}\right)_{w} \frac{\partial T}{\partial x}-k\left(\frac{\partial^{2} T}{\partial x^{2}}+\frac{\partial^{2} T}{\partial y^{2}}\right)=0
$$

Where $T$ represents the average temperature of the porous medium; $t$ is the operation time; $k$ is the bulk thermal conductivity, and $\rho C_{p}$ represents the volumetric heat capacity of the porous medium. If $\eta$ is the soil porosity, 
the bulk properties can be expressed as Eq.(2).

$$
k=\eta k_{f}+(1-\eta) k_{s}, \quad \rho C_{p}=\eta\left(\rho C_{p}\right)_{f}+(1-\eta)\left(\rho C_{p}\right)_{s}, a=k / \rho C_{p}
$$

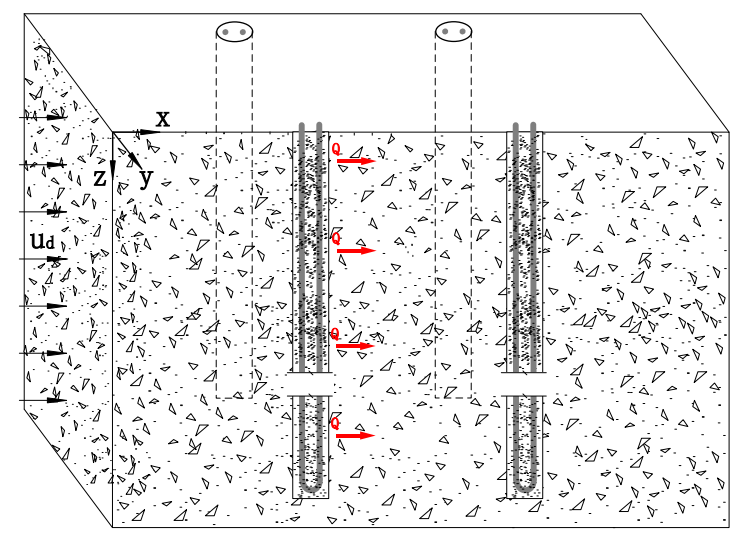

Fig. 1. Schematic of the vertical BHEs with groundwater flow ( $Q$ is the heating and cooling loads)

In the field application, the diameter of the borehole ranges from 0.1 to $0.2 \mathrm{~m}$ and the depth normally varies from 40 to $200 \mathrm{~m}$. The aspect ratio between the depth and the diameter of the borehole is high and the borehole can be considered as a line heat source. According to the infinite line-source (ILS) model, the G-function at $r=$ $r_{b}$ due to a unit-step load is shown in Eq. (3) (Eskilson and Claesson 1988).

$$
G_{I L S}(t)=\frac{1}{4 \pi k} \int_{\frac{r_{b}^{2}}{4 a t}}^{\infty} \frac{\exp (-u)}{u} d u=\frac{1}{4 \pi k} E_{1}\left(\frac{r_{b}^{2}}{4 a t}\right)
$$

Where $u$ is an integral variable; $E_{1}$ is an exponential integral function. In many cases the heating loads are not in balance with the cooling loads on a year-round basis, and the excessive load will accumulate in the ground and thus the effect of the heat transfer on the ground surface should be taken into account. Research showed that after 1.6 years, the ILS model would produce large error due to the neglect on the axial heat conduction of the borehole (Philippe, et al. 2009), and this would increase the length of BHEs by 15\% during the initial design (Marcotte, et al. 2010). This limitation can be addressed by the finite line-source (FLS) model, which is derived from the method of images of heat sources. The G-function at $r=r_{b}$ due to a unit-step load can be expressed by Equation (4) (Zeng, et al. 2002).

$$
G_{F L S}(t)=\frac{1}{4 \pi H k} \int_{0}^{\mathrm{H}} d z \int_{0}^{\mathrm{H}}\left[\frac{\operatorname{erfc}\left(\frac{\sqrt{r_{b}^{2}+(z-l)^{2}}}{2 \sqrt{a t}}\right)}{\sqrt{r_{b}^{2}+(z-l)^{2}}}-\frac{\operatorname{erfc}\left(\frac{\sqrt{r_{b}^{2}+(z+l)^{2}}}{2 \sqrt{a t}}\right)}{\sqrt{r_{b}^{2}+(z+l)^{2}}}\right] d l
$$

Where $l$ and $z$ denote the integral variables, and $\operatorname{erfc}(x)$ is the complementary error function. It should be noted that both ILS and FLS models are based on the assumptions of steady heat conduction in borehole. Therefore, they cannot be used directly for short time-step analysis in vertical BHEs. As a substitute, the composite-medium line-source (CMLS) theory ( $\mathrm{Li}$ and Lai 2012) has been proposed to include the transient thermal process in the borehole and to further investigate the effect caused by the difference in the thermal properties of the ground and the backfilling material. As indicated in Fig. 2, the U-shaped tube is treated as two 
infinite heating lines, and the wall temperature of the U-shaped tube is considered as characteristic temperature of the model and this value is the average of temperatures at points A and B. The short-term G-function for BHEs with single U-shaped tube is defined in Equations (5) and (6) (Li, et al. 2014; Yang and Li 2014).

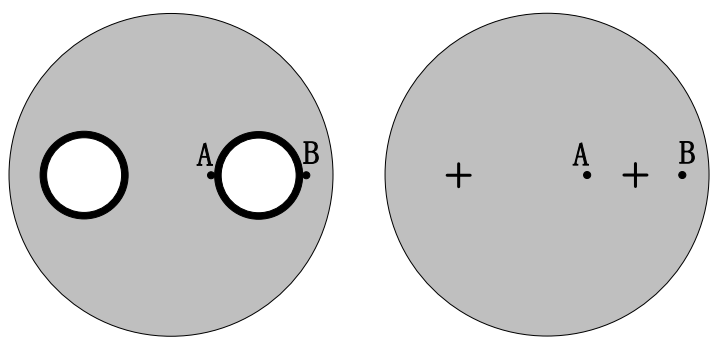

Fig. 2. The schematic of CMFL model for single U-shaped tube in the borehole

$$
\begin{aligned}
& G_{C M L S}(t)=\frac{1}{2 \pi k_{b}} \sum_{n=-\infty}^{+\infty} \int_{0}^{+\infty}\left[1-\exp \left(-u^{2} a_{b} t\right)\right] \times \frac{J_{n}\left(u r^{\prime}\right)(\varphi \mathrm{g}-\psi f)\left[J_{n}\left(u r_{A}\right)+J_{n}\left(u r_{B}\right)\right]}{2 u\left(\varphi^{2}+\psi^{2}\right)} d u \\
& \varphi=a^{*} k^{*} J_{n}\left(u r_{b}\right) J_{n}^{\prime}\left(a^{*} u\right)-J_{n}^{\prime}\left(u r_{b}\right) J_{n}\left(a^{*} u\right) \\
& \psi=a^{*} k^{*} J_{n}\left(u r_{b}\right) Y_{n}^{\prime}\left(a^{*} u\right)-J_{n}^{\prime}\left(u r_{b}\right) Y_{n}\left(a^{*} u\right) \\
& f=a^{*} k^{*} Y_{n}\left(u r_{b}\right) J_{n}^{\prime}\left(a^{*} u\right)-Y_{n}^{\prime}\left(u r_{b}\right) J_{n}\left(a^{*} u\right) \\
& \mathrm{g}=a^{*} k^{*} Y_{n}\left(u r_{b}\right) Y_{n}^{\prime}\left(a^{*} u\right)-Y_{n}^{\prime}\left(u r_{b}\right) Y_{n}\left(a^{*} u\right)
\end{aligned}
$$

Where $a^{*}=\sqrt{a_{b} / a}$ and $k^{*}=k / k_{b}$ are the dimensionless variables; $r^{\prime}$ is the radius position of the heat line-sources (Fig. 2); $r_{A}$ and $r_{B}$ are the radius coordinates of points $\mathrm{A}$ and $\mathrm{B}$ respectively; $u$ is the integral variable.

In the cases with groundwater flow, when neglect the effects of thermal dispersion, moving infinite line heat source (MILS) and moving finite line heat source (MFLS) models are the two main methods that take into account of the groundwater effect. (Chiasson and Connell 2011) examines two other analytical solutions for the heat transfer characteristics around closed-loop borehole heat exchangers in significant groundwater flow and proposes a model could account for the effects of thermal dispersion with groundwater advection. In MILS model, by considering the dimensionless parameters $(P e$ and $F o$ ) and defining the integral average temperature of a circle with given radius, the G-function at $r=r_{b}$ due to a unit-step load is given in Eq. (7) (Diao, et al. 2004).

$$
G_{M I L S}(t)=\frac{1}{4 \pi k} \int_{0}^{\pi}\left\{\exp \left[\frac{P e}{2} R^{\prime} \cos (\varphi)\right] \int_{0}^{P e^{2} F o / 4} \frac{1}{\psi} \exp \left[-\psi-\frac{P e^{2} R^{\prime 2}}{16 \psi}\right] d \psi\right\} d \varphi
$$

Where the reference length for non-dimensional parameters is $H, R^{\prime}=\sqrt{x^{2}+y^{2}} / H, R=\sqrt{{R^{\prime}}^{2}+\left(Z-Z^{\prime}\right)^{2}}, Z=$ $z / H, Z^{\prime}=z^{\prime} / H$ are the dimensionless parameter; $\psi$ is the integral variable. By considering the impact of the axial heat transfer in BHEs, MFLS model was developed with the G-function shown in Eqs. (8) and (9) (Molina-Giraldo, et al. 2011). Eq. (8) can be solved directly using numerical integral method, or it can be calculated by the simplified method given in the reference (Lamarche and Beauchamp 2007).

$$
\begin{gathered}
G_{M F L S}(t)=\frac{1}{4 \pi k} \int_{0}^{1} d Z \int_{0}^{\pi}\left\{2 \exp \left[\frac{P e}{2} R^{\prime} \cos (\varphi)\right]\left[\int_{0}^{1} f(R, F o, P e) d Z^{\prime}-\int_{-1}^{0} f(R, F o, P e) d Z^{\prime}\right]\right\} d \varphi \\
f(R, F o, P e)=\frac{1}{4 R}\left[\exp \left(-\frac{P e}{2} R\right) \operatorname{erfc}\left(\frac{R-P e F o}{2 \sqrt{F o}}\right)+\exp \left(\frac{P e}{2} R\right) \operatorname{erfc}\left(\frac{R+P e F o}{2 \sqrt{F o}}\right)\right]
\end{gathered}
$$

\section{MPROVED FULL-SCALE ANALYTI CAL MODEL}

Fig.3 indicates the temperature profiles in one single borehole predicted by CMLS, ILS, FLS and MFLS 
models. The time scale ranges from 0.5 min to 95 years and it can be roughly divided into two subintervals by a specific transition time $t_{b}$. This transition time is related to the structure of the borehole and it can be estimated by following a scaling relation $t_{b} \approx\left(2 r_{b}\right)^{2} / a_{b}$ (Li and Lai 2015). The time scale is divided into short-term (0-5 hours) and medium-/long-term ( $>5$ hours). When there is no groundwater flow or the effect of water flow can be neglected, another transition time $t_{H} \approx 70$ days (estimated from Fig.3) is used and $t_{H}$ is related to the length of the borehole. It should be noted that these transition times are pre-required in the current full-scale models and the solutions are derived by shifting the line source solutions at an appropriate "breaking time".

As reviewed in the previous section, the traditional FLS model is able to be used in the mid-/long-term analysis and CMLS model is preferred in the short-term without considering the groundwater effect. Both MILS and MFLS models include the advection effect, but they would only be appropriate for the long-term. Therefore, a combination of CMLS and MFLS models would be able to include the effects of full time scale and advection caused by the water flow. By following a similar idea of "matching asymptotic expansion" proposed by other researchers (Li, et al. 2014 Javed 2011), the G-function of an improved CMLS-MFLS model is expressed as Eq. (10), where $G_{C M L S}(t), G_{M F L S}(t)$ and $G_{M I L S}(t)$ can be determined from Eqs. (5) to (9). When there is no effect of groundwater flow, the G-function Eq. (10) can also be written as the Eq. (11). It should be noted that different from other full-scale models, the transition times are not required to determine the improved G-function.

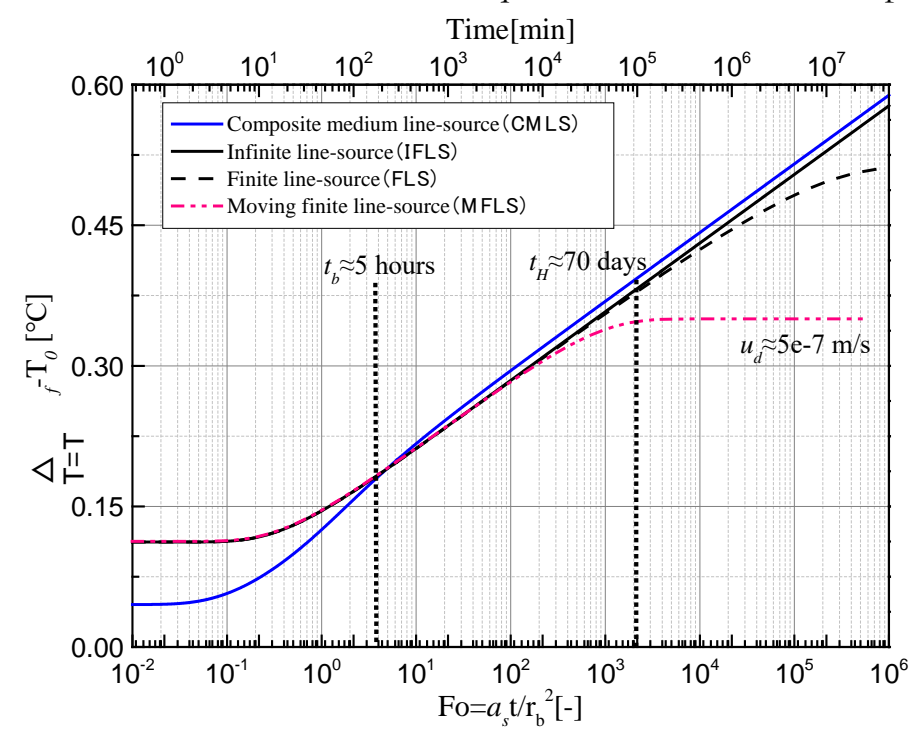

Fig.3. Temperature response of the circulating fluid in a borehole due to a unit-step thermal load, which is the solution of various analytical models (detailed input parameters are provided in Table 1)

$$
\begin{gathered}
G_{C M L S-M F L S}(t)=G_{C M L S}(t)+G_{M F L S}(t)-G_{M I L S}(t)=\frac{1}{2 \pi k_{b}} \sum_{n=-\infty}^{+\infty} \int_{0}^{+\infty}\left[1-\exp \left(-u^{2} a_{b} t\right)\right] \times \\
\frac{J_{n}\left(u r^{\prime}\right)(\varphi \mathrm{g}-\psi f)\left[J_{n}\left(u r_{A}\right)+J_{n}\left(u r_{B}\right)\right]}{2 u\left(\varphi^{2}+\psi^{2}\right)} d u+\frac{1}{4 \pi k}\left\{\int_{0}^{1} d Z \int_{0}^{\pi}\left\{2 \exp \left[\frac{P e}{2} R^{\prime} \cos (\varphi)\right] \int_{-1}^{1} f(R, F o, P e) d Z^{\prime}\right\} d \varphi-\right. \\
\left.\int_{0}^{\pi}\left\{\exp \left[\frac{P e}{2} R^{\prime} \cos (\varphi)\right] \int_{0}^{P e^{2} F o / 4} \frac{1}{\psi} \exp \left[-\psi-\frac{P e^{2} R^{\prime 2}}{16 \psi}\right] d \psi\right\}\right\}
\end{gathered}
$$




$$
\begin{gathered}
G_{C M L S-M F L S}(t)=G_{C M L S}(t)+G_{F L S}(t)-G_{I L S}(t)=\frac{1}{2 \pi k_{b}} \sum_{n=-\infty}^{+\infty} \int_{0}^{+\infty}\left[1-\exp \left(-u^{2} a_{b} t\right)\right] \times \\
\frac{J_{n}\left(u r^{\prime}\right)(\varphi \mathrm{g}-\psi f)\left[J_{n}\left(u r_{A}\right)+J_{n}\left(u r_{B}\right)\right]}{2 u\left(\varphi^{2}+\psi^{2}\right)} d u+\frac{1}{4 \pi H k} \int_{0}^{\mathrm{H}} d z \int_{0}^{\mathrm{H}}\left[\frac{\operatorname{erfc}\left(\frac{\sqrt{r_{b}^{2}+(z-l)^{2}}}{2 \sqrt{a t}}\right)}{\sqrt{r_{b}^{2}+(z-l)^{2}}}-\frac{\operatorname{erfc}\left(\frac{\sqrt{r_{b}^{2}+(z+l)^{2}}}{2 \sqrt{a t}}\right)}{\sqrt{r_{b}^{2}+(z+l)^{2}}}\right] d l-\frac{1}{4 \pi k} E_{1}\left(\frac{r_{b}^{2}}{4 a t}\right)
\end{gathered}
$$

The temperature of the circulating fluid due to a unit-step heating/cooling load can be obtained as Eq. (12).

$$
T_{f}(t)-T_{S, 0}=q_{l}\left[G_{C M L S-M F L S}(t)+R_{p}\right]
$$

Where $q_{l}$ is the heat flux per unit length, $R_{p}=R_{\text {conv }}^{\prime}+R_{\text {cond }}^{\prime}$ is the U-shaped pipe thermal resistance, $R_{\text {conv }}^{\prime}=$ $1 /\left(4 \pi r_{o} h\right)$ is the convective resistance and $R_{\text {cond }}^{\prime}=\ln \left(r_{o} / r_{i}\right) /\left(4 \pi k_{p}\right)$ is the conductive resistance of the U-shaped tube. The other important thing need to declare is that the steady-state borehole thermal resistance $R_{b}=R_{\text {conv }}^{\prime}+R_{\text {cond }}^{\prime}+R_{b}^{\prime}=R_{p}+R_{b}^{\prime}$ is used in the section, where $R_{b}^{\prime}$ is the thermal resistance of the grout. According to the literature (Lamarche, et al. 2010), $R_{b}^{\prime}$ is best predicted by the analytical solution derived from multipole method, and this expression is included in the CMLS-MFLS model and other formulates.

\section{MODEL VALI DATION AND DISCUSSI ON}

Numerical solutions by finite difference method or finite element method are commonly used to solve heat conduction problems. It is more flexible to deal with complicated geometries or other complexities such as the case of compound medium. In this section, a 2-D model was built to simulate the transient behavior of the thermal response in the actual U-shaped tube, which is normally in the short and medium time scale. Because the axial heat conduction has a significant effect only in a long time scale, the axial heat transfer is not considered and the solutions would be used to validate the new analytical solution developed in the previous section.

Fig. 4 indicates the 2-D mesh used in the finite element simulations. Constant heat flux $(0.5 \mathrm{~W} / \mathrm{m})$ were applied uniformly on the exterior surfaces of the two inner tubes. The infinite domain was replaced by a finite region with a large radius $(10 \mathrm{~m})$, and the initial temperatures of both the soil and the outer boundaries were assumed as constant $\left(15^{\circ} \mathrm{C}\right)$. The characteristic temperature of the tube walls equal to the average temperature of the circle as shown in the figure. Table 1 lists the physical properties of the medium and the operating conditions of the unsteady heat transfer procedure.

Fig.5 compares the predicted fluid temperature rise obtained from both analytical and numerical models. It is observed that the improved full-scale model (CMLS-MFLS) matched best with the numerical solutions in the short- and medium-term. The temperature rises also followed the variation trend of FLS model in the long-term. It should be noted that the traditional line-source models required to shift the temperature response after the transition time $t_{b}$. Therefore, the solution of the proposed CMLS-MFLS model would not rely on the transition time and could predict the temperature profile of the fluid in the tube of the borehole in the entire time scale. 


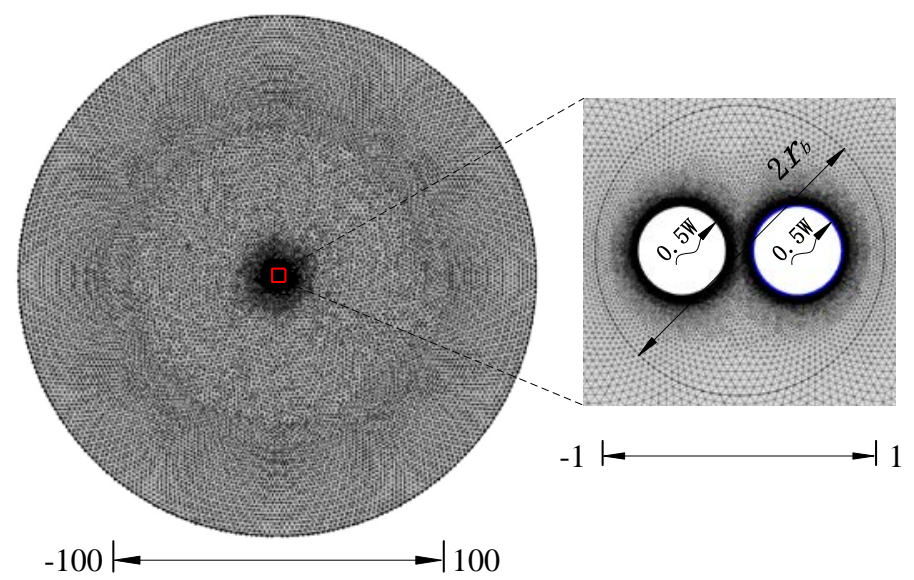

Fig. 4. Computation mesh used in the finite element simulations (without the U-shaped tube); the scale at the bottom indicates a non-dimensional distance based on the borehole radius

Table 1. Physical properties, and operating conditions of single U-tube BHEs models

\begin{tabular}{cccc}
\hline Properties & Values & Properties & Values \\
\hline \hline$q_{l}$ & $1 \mathrm{~W} / \mathrm{m}$ & $r_{b}$ & $0.065 \mathrm{~m}$ \\
$k_{p}$ & $0.42 \mathrm{~W} /(\mathrm{mK})$ & $\mathrm{H}$ & $100 \mathrm{~m}$ \\
$a$ & $1.33 \times 10^{-6} \mathrm{~m}^{2} / \mathrm{s}$ & $\mathrm{x}_{U}$ & $0.026 \mathrm{~m}$ \\
$a_{b}$ & $4.84 \times 10^{-7} \mathrm{~m}^{2} / \mathrm{s}$ & $r_{i}$ & $0.017 \mathrm{~m}$ \\
$T_{0}$ & $15^{\circ} \mathrm{C}$ & $r_{o}$ & $0.02 \mathrm{~m}$ \\
\hline
\end{tabular}

Fig. 6 compares the thermal response curves derived with groundwater flow. Similar to the previous findings, the proposed CMLS-MFLS model could better predict the temperature rise in the short-term than the traditional MFLS model. In order to further investigate the impact of water flow on the thermal response, both CMLS-MFLS and CMLS-MILS models were compared at different flow rates. Results showed that the differences of fluid temperature obtained from moving finite and infinite line-source models were gradually decrease with the velocity increased. When Pe was higher than 20, the solutions derived from CMLS-MFLS and CMLS-MILS were almost overlapped, which indicated that the effect of axial heat transfer was weakened by the enhanced convection and the axial heat conduction in the borehole could almost be neglected. However, when Pe was less than 10, the results temperature rises predicted by CMLS-MFLS almost matched with the results in FLS, which indicated that the effect of groundwater could be neglected, and the axial heat conduction in the borehole would be the main factor to affect the long-term response.

\section{CONCLUSION}

A new full-scale CMLS-MFLS model, which could account for the entire time scale and the effect of groundwater flow, was proposed in this paper to predict the thermal response of a single, vertical BHEs. The analytical solution was validated by a numerical solution and compared with other typical analytical models. The analytical solutions of the proposed model remained simple in the expression and were convenient to use in the design of BHEs. The effect of axial heat loss and underground water flow were analyzed based on the proposed model. It was observed that when Pe was high (>20 in this study), the axial heat loss had a minor impact on the thermal response and could almost be neglected. When Pe was low ( $<10$ in this study), the axial heat loss would be 
the main factor in the long-term response and the advection effect could be neglected.

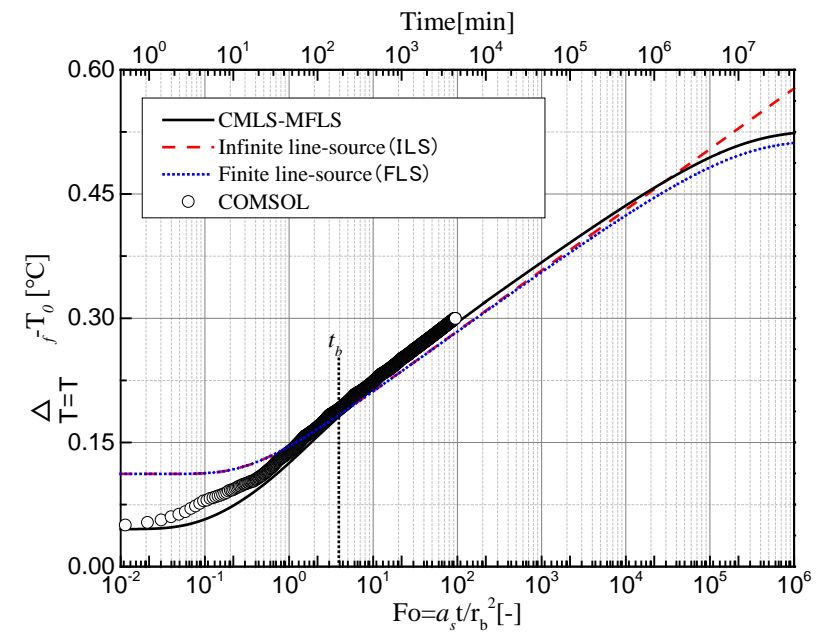

Fig.5. Validation of the new CMLS-MFLS model with a finite element method when $\mathrm{Pe}=0$

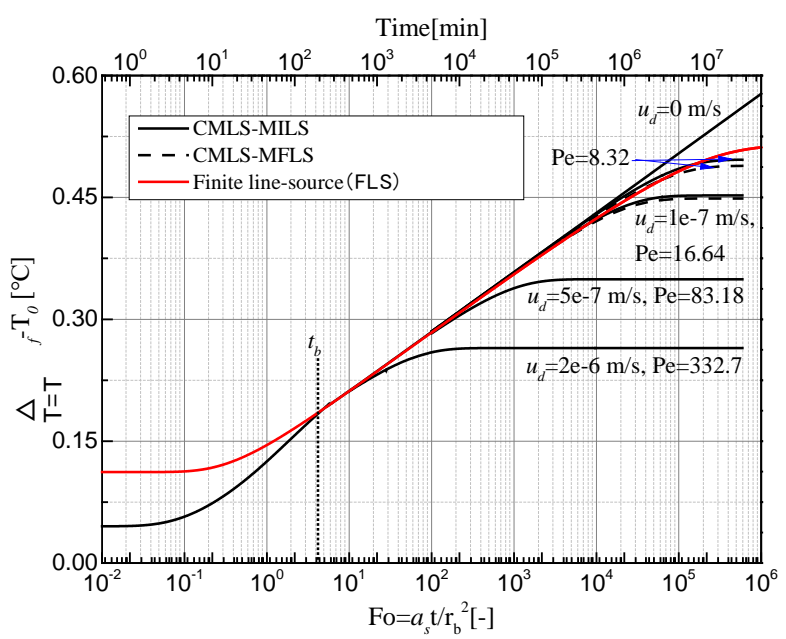

Fig. 6. Thermal response curves of CMLS-MFLS model at different groundwater flow rates

\section{ACKNOWLEDGMENTS}

The authors would like to thank and acknowledge Natural Science Foundation Grant No. 51706078 and Hubei Natural Science Foundation Grant No. 2017CFB131 for funding and supporting this work. The authors would also give special thanks to Dr. Min Li for providing the original simulation codes of CMLS model and Dr. Zhenhua Chai for his tremendous help on the mathematics.

\section{NOMENCLATURE}
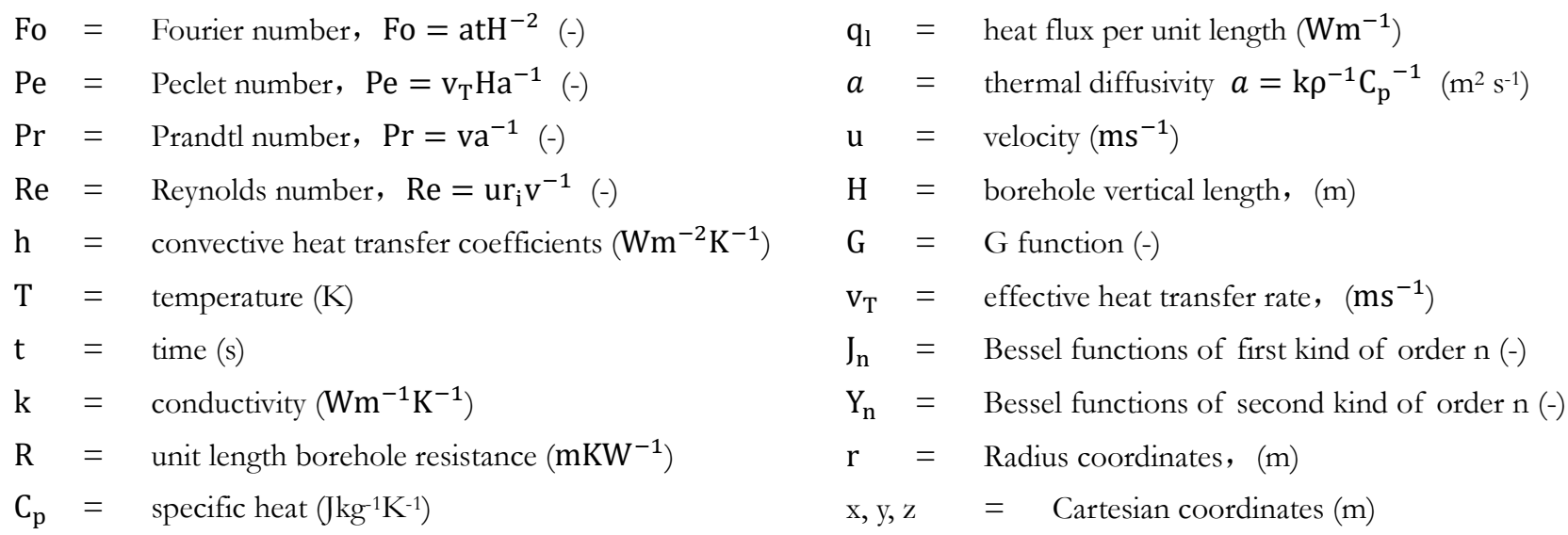

\section{Greek letters}

$\varphi=$ angle with $\mathrm{x}$-axis $(\mathrm{rad})$

$\eta \quad=\quad$ ground porosity $(-)$

$\rho=$ density $\left(\mathrm{kgm}^{-3}\right)$

\section{Subscripts and superscripts}

$I=$ internal pipe

$b=$ grout

$f \quad=$ fluid $s \quad=$ solid

$p=$ pipe

$0=$ external pipe
$U=$ shank spacing

' $=$ dimensionless

$d=$ Darcy 


\section{REFERENCES}

Capozza, A. and M.D. Carli, et al. 2013. Investigations on the influence of aquifers on the ground temperature in ground-source heat pump operation. Applied Energy 107:350-363.

Chiasson, A. and A.O. Connell. 2011. New analytical solution for sizing vertical borehole ground heat exchangers in environments with significant groundwater flow: parameter estimation from thermal response test data. Hvac \& R Research 17:1000-1011.

Claesson, J. and G. Hellström. 2011. Multipole method to calculate borehole thermal resistances in a borehole heat exchanger. Hvac \& R Research 17:895-911.

Diao, N. and Q. Li, et al. 2004. Heat transfer in ground heat exchangers with groundwater advection. International Journal of Thermal Sciences 43:1203-1211.

Diao, N.R. and H.Y. Zeng, et al. 2004. Improvement in modeling of heat transfer in vertical ground heat exchangers. Hvac \& R Research 10:459-470.

Eskilson, P. and J. Claesson. 1988. Simulation model for thermally interacting heat extraction boreholes. Numerical Heat Transfer Applications 13:149-165.

Hellström, G. 1991. Ground heat storage: thermal analyses of duct storage systems. Department of Mathematical Physics University of Lund, Sweden.

Javed, S. 2011. An analytical method to calculate borehole fluid temperatures for time-scales from minutes to decades. ASHRAE Transactions 117:279-288.

Javed, S. and J. Claesson. 2011. New analytical and numerical solutions for the short-term analysis of vertical ground heat exchangers. ASHRAE Transactions 117:3-12.

Kim, E.J. and J.J. Roux, et al. 2010. Numerical modelling of geothermal vertical heat exchangers for the short time analysis using the state model size reduction technique. Applied Thermal Engineering 30:706-714.

Lamarche, L. and B. Beauchamp. 2007. A new contribution to the finite line-source model for geothermal boreholes. Energy \& Buildings 39:188-198.

Lamarche, L. and S. Kajl, et al. 2010. A review of methods to evaluate borehole thermal resistances in geothermal beat-pump systems. Geothermics 39:187-200.

Li, M. and A.C.K. Lai. 2012. New temperature response functions (g functions) for pile and borehole ground beat exchangers based on composite-medium line-source theory. Energy 38:255-263.

Li, M. and A.C.K. Lai. 2015. Review of analytical models for heat transfer by vertical ground heat exchangers (ghes): a perspective of time and space scales. Applied Energy 151:178-191.

Li, M. and P. Li, et al. 2014. Full-scale temperature response function (g-function) for heat transfer by borehole ground heat exchangers (ghes) from sub-hour to decades. Applied Energy 136:197 - 205.

Marcotte, D. and P. Pasquier, et al. 2010. The importance of axial effects for borehole design of geothermal heat-pump systems. Renewable Energy 35:763-770.

Molina-Giraldo, N. and P. Blum, et al. 2011. A moving finite line source model to simulate borehole beat exchangers with groundwater advection. International Journal of Thermal Sciences 50:2506-2513.

Nield, D.A. and A. Bejan. 2006. Convection in porous media. Springer.

Philippe, M. and M. Bernier, et al. 2009. Validity ranges of three analytical solutions to heat transfer in the vicinity of single boreholes. Geothermics 38:407-413.

Saner, D. and R. Juraske, et al. 2010. Is it only $\mathrm{co}_{2}$ that matters? A life cycle perspective on shallow geothermal systems. Renewable \& Sustainable Energy Reviews 14:1798-1813.

Somogyi, V. and V. Sebestyén, et al. 2017. Scientific achievements and regulation of shallow geothermal systems in six European 
countries - a review. Renewable \& Sustainable Energy Reviews 68:934-952.

Yang, Y. and M. Li. 2014. Short-time performance of composite-medium line-source model for predicting responses of ground heat exchangers with single u-shaped tube. International Journal of Thermal Sciences 82:130 - 137.

Yavuzturk, C. 1999. Modeling of vertical ground loop heat exchangers for ground source heat pump systems. Oklahoma State University, Stillwater, Oklahoma, U.S.A.

Zeng, H.Y. and N.R. Diao, et al. 2002. A finite line-source model for boreholes in geothermal heat exchangers. Heat Transfer-Asian Research 31:558-567. 